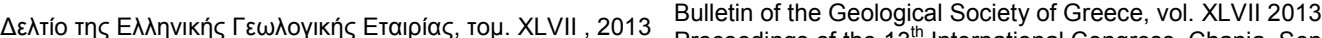
$\triangle 1$ Proceedings of the $13^{\text {th }}$ International Congress, Chania, Sept.

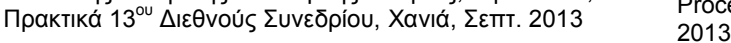

\title{
RECONSTRUCTING CONCEALED CULTURAL REMAINS THROUGH INTEGRATED GEOPHYSICAL TOMOGRAPHIC METHODS
}

\author{
Soupios P. ${ }^{1}$, Papadopoulos N. ${ }^{2}$ and Sarris A. ${ }^{2}$ \\ ${ }^{I}$ Department of Natural Resources \& Environment, Technological Educational Institute of Crete, \\ Greece,email: soupios@chania.teicrete.gr \\ ${ }^{2}$ Laboratory of Geophysical-Satellite Remote Sensing \& Archaeo-environment, Institute for \\ Mediterranean Studies - Foundation for Research \& Technology, Hellas (IMS/FORTH), emails: \\ nikos@ims.forth.gr,asaris@ret.forthnet.gr.
}

\begin{abstract}
The purpose of this work was to compare the mapping of shallow subsurface archaeological structures through Seismic Refraction Tomography (SRT), Electrical Resistivity Tomography (ERT) and Ground Penetrating Radar (GPR) methods. For achieving the goals of the project, a specific section of the archaeological site in Delphi has been surveyed through the employment of the above techniques. For the SRT survey, twenty four P-wave geophones were installed randomly in a 50 by $40 \mathrm{~m}$ area. Totally seventy three (73) shots were made by striking a metal plate with a sledgehammer to collect about 1752 travel-times. The pole-dipole array was employed to capture the ERT data along twenty densely spaced parallel profiles. The GPR data were collected along parallel sections with a resolution of $50 \mathrm{~cm}$ between the lines. The SRT and ERT field data were processed with modern tomographic inversion algorithms for the reconstruction of the 3-D velocity and resistivity models describing the buried archaeological remains and the subsurface matrix up to the depth of 5 meters below the ground surface. GPR signals were enhanced with specific filters signifying the shallow structures up to 2 meters below the ground surface. The integrated processing results indicate the existence of walls buried in a relatively uniform background soil. The outcome of this approach signifies that SRT, $E R T$ and GPR methods can be used as a validation tool in any archaeological investigation by providing accurate tomographic subsurface models and contribute in cultural resources management.
\end{abstract}

Key words: resistivity tomography, seismic refraction tomography, $G P R, 3 D$, Delphi.

\section{Пєрí $\eta \psi \eta$}

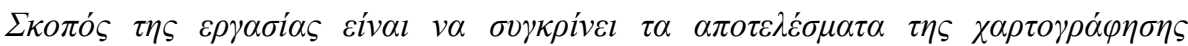

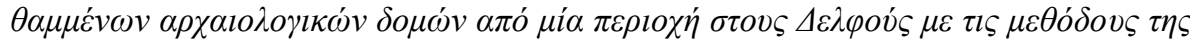

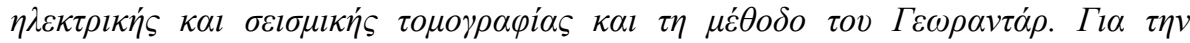

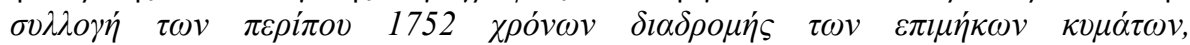

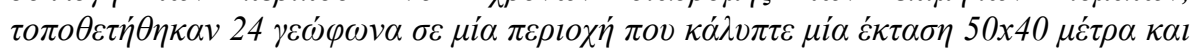

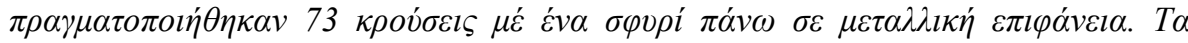

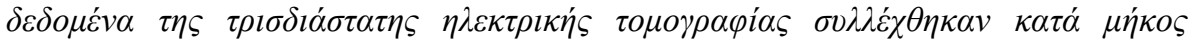

$\underline{\text { XLVII, No } 3-1260}$ 


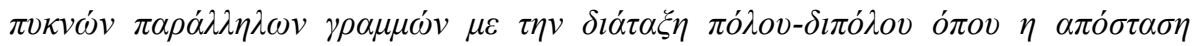

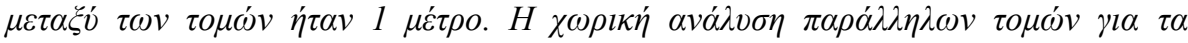

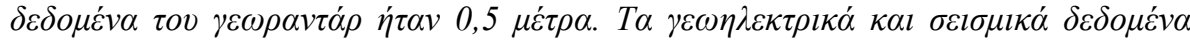

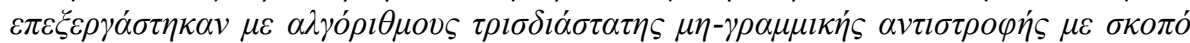

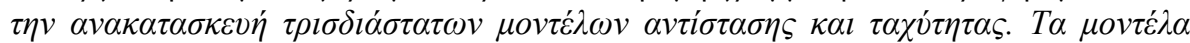

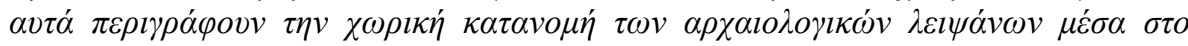

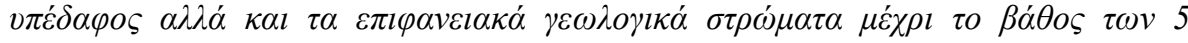

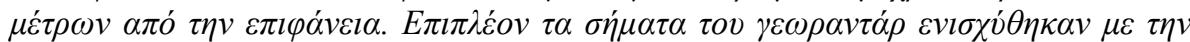
$\varepsilon \varphi \alpha \rho \mu о \gamma \eta \dot{~ \sigma v \gamma \kappa \varepsilon \kappa \rho l \mu \varepsilon ́ v \omega v ~ \varphi i ́ \lambda \tau \rho \omega v ~ \mu \varepsilon ~ \sigma \kappa o \pi o ́ ~ \tau \eta v ~ \varepsilon v i ́ \sigma \chi v \sigma \eta ~ \tau \eta \varsigma ~ \alpha \rho \chi \alpha l o \lambda o \gamma l \kappa \eta ́ s ~}$

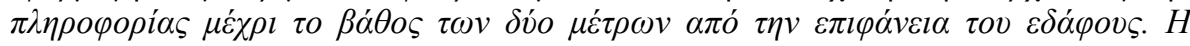

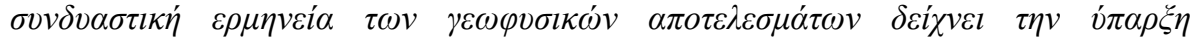

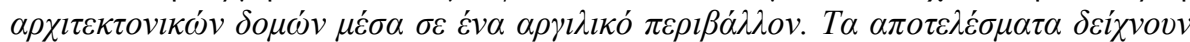

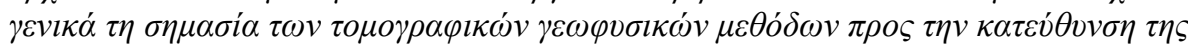

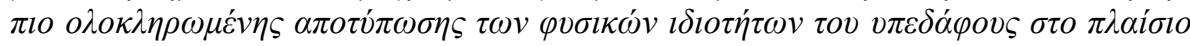

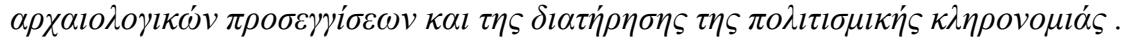

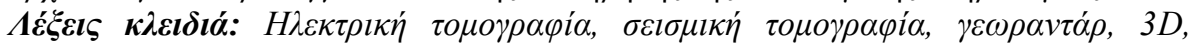
$\Delta \varepsilon \lambda \varphi o i$.

\section{Introduction}

Delphi is both an archaeological site and a modern town in lower central Greece on the southwestern side of Mount Parnassus. The site is well known for its Delphic oracle, the most important oracle in the classical Greek world, and a major site for the worship of the god Apollo. The Pythian Games were held every four years, starting in 776 BC. The first excavations in Delphi started in 1892 with the excavation to the sanctuary of Apollon to initiate in 1887. Excavations required the displacement of the old medieval village of Kastri, which was built on the top of the archaeological site, to the outer limits of the site, where now the modern town of Delphi is located. Below the soil depositions due to landslides, the sanctuary of Apollon, the Gymnasium, the Stadium, the settlement and its cemeteries started to be revealed.

In 2012, a three-year archaeological mission was initiated by the French School of Athens having three components (Luce 2012). Through the cleaning of the 19th century trenches, the topographic mapping of the surface monuments and the architectural relics, and the geophysical mapping of the area, the archaeological campaign aims to study the urban organization of the city and its development since the beginning of the occupation in the $16^{\text {th }}$ century $\mathrm{BC}$ to its abandonment in the $7^{\text {th }}$ century AD. The first phase of the geophysical investigations was completed within July 2012 focussing mainly at the north-west section of the archaeological site. In this particular area the archaeologists of the $19^{\text {th }}$ century opened a series of about 30 roughly parallel exploratory trenches that brought to light a number of architectural remains. The goals of the geophysical campaign were to reveal the plan of structures between the trenches, with particular attention to those who are continuing from the open ones.

The steep slopes of the terrain of Delphi $(\sim 30-40 \%)$, the wooded areas, the fire-fighting hoses, the various metal fragments distributed randomly around the site and the deep old trenches made the geophysical prospection of Delphi a real challenge. In order to maximize the results of the geophysical prospection and test the quality of the signals collected, five methods were applied in the site: magnetic gradiometry, electrical resistivity tomography (ERT), multi-frequency controlled source electromagnetic (CSEM), ground penetrating radar (GPR) and seismic refraction tomography (SRT). The investigations were focused below the road that leads to the stadium and above the main road that leads to the sanctuary. The layout of the geophysical grids and the locations of the geophones or the resistivity tomography transects was carried out by a total station and a differential GPS survey (Figure 1).

XLVII, No 3 - 1261 
This work will focus on the results of an integrated geophysical approach that was applied in a small section of the site. This 50 by 40 meters area is outlined with the black thick rectangle in Figure 1. The plateau and the road that exist close to the facilities of the performances of the theatre and the storage area of epigraphic stones found in the site were scanned through by seismic refraction and resistivity tomography methods in order to enhance the information context up to the depth of 5 meters below the ground surface. A complementary GPR survey was used to highlight the superficial structures up to 2 meters. These geophysical techniques were the only ones allowed in the area, as the rest of the methods could not have been employed due to the modern metallic structures that existed in the area. In the following paragraphs the field strategy, the processing steps and the results by the integrated interpretation are explained.

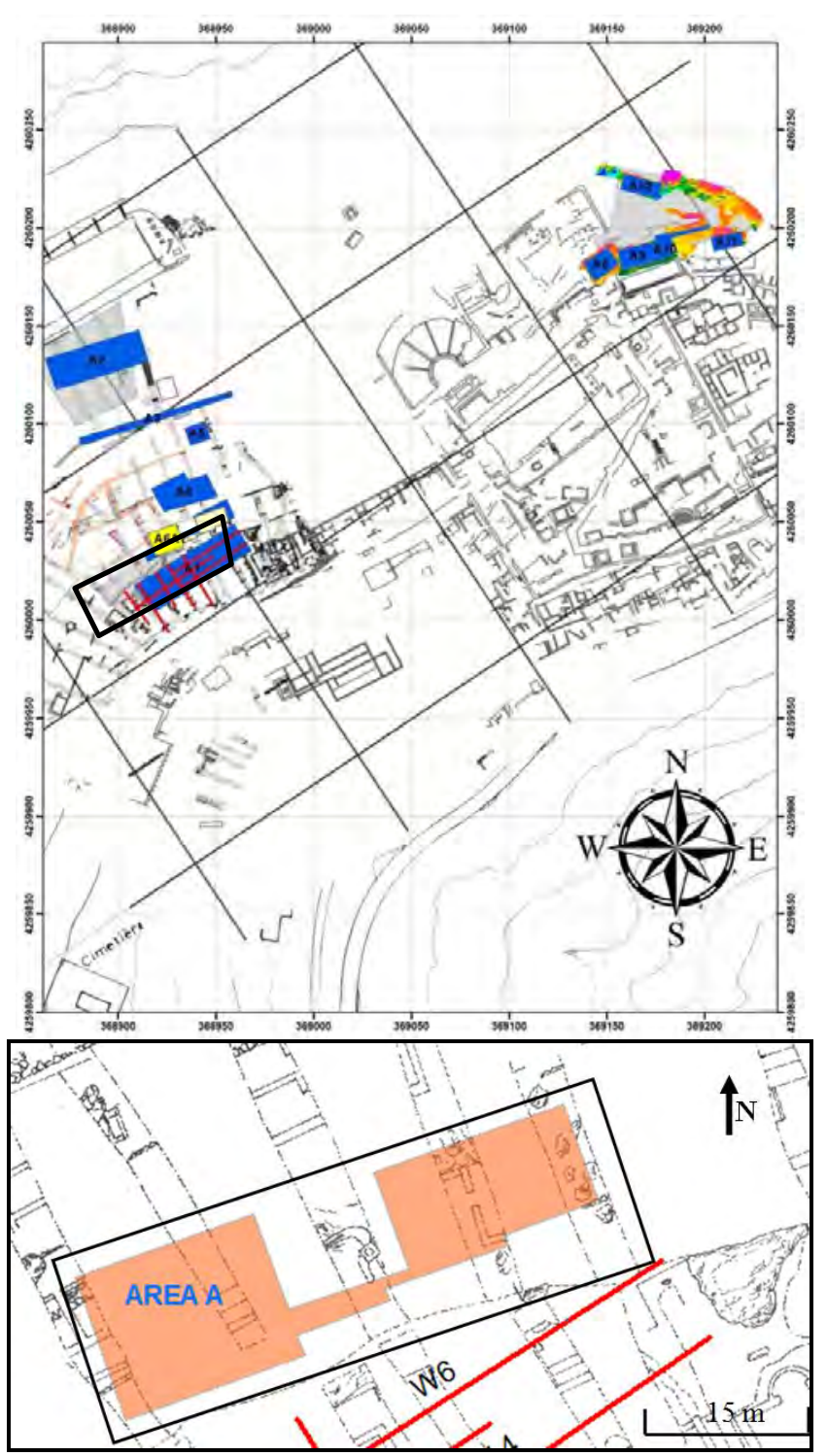

Figure 1 - Overlay of the geophysical grids and transects from the 2012 survey in the area of investigation as it is depicted by the topographic plan of Delphi. The blue areas were all scanned with the GPR. The red lines denote the individual 2-D ERT profiles. The thick rectangular depicts the section (Area A) where the experimental 3-D ERT, SRT and GPR techniques were employed.

XLVII, No $3-1262$ 


\section{Data Collection and Processing}

\subsection{Electrical Resistivity Tomography}

The specific scheme of collecting measurements within a dense network of parallel 2-D ERT sections was followed (Papadopoulos et al., 2006) in order to survey an area of about 400 square meters, excluding the inaccessible parts due to modern constructions that prohibited the employment of electrodes in these cases. The basic inter-electrode interval along each profile and the inter-line spacing was one meter $(a=1 \mathrm{~m})$. The lines had variable length due to obstacles that were scattered in the area. A protocol of a sequence of measurements employing the pole-dipole configuration was programmed into the Syscal Pro Switch resistivity instrument in order to capture the apparent resistivity data with maximum $\mathrm{Nsep}=10$. Additional data with multiple "a" spacing (1a, 2a) were also collected to enhance the signal to noise ratio while trying to investigate at larger depths. Figure 2 (center) shows details of the instrumentation during the collection of the ERT data. The pre-processing stages of the ERT data include the geometry correction of the individual lines and the removal of erroneous extreme high or low apparent resistivity values through despiking filters.

After cleaning the data and bringing them to a suitable format an iterative inversion algorithm (Papadopoulos et al., 2011) was used to reconstruct a 3-D resistivity model of the site. The algorithm uses a 3-D Finite Element Method scheme to numerically solve the Poisson's equation and the adjoint equation technique to calculate the sensitivity matrix. The augmented system $\left[\begin{array}{c}\mathbf{J}_{\mathrm{k}} \\ \sqrt{\lambda_{\mathrm{k}}} \mathbf{C}\end{array}\right] \cdot \mathbf{d} \mathbf{x}_{\mathrm{k}}=\left[\begin{array}{l}\mathbf{d} \mathbf{y}_{\mathrm{k}} \\ \mathbf{0}\end{array}\right]$ is solved with an iterative solver (LSMR-Fong and Saunders 2011), where $\mathrm{k}$ is the iteration number, $\mathbf{J}_{\mathrm{k}}$ is the Jacobian matrix estimate of the $\mathbf{x}_{\mathrm{k}}$ resistivity distribution, $\mathbf{d} \mathbf{x}_{\mathrm{k}}$ is the resistivity correction vector, $\mathbf{d} \mathbf{y}_{\mathrm{k}}=\mathbf{y}-\mathbf{F}\left(\mathbf{x}_{\mathrm{k}}\right)$, where $\mathbf{F}\left(\mathbf{x}_{\mathrm{k}}\right)$ is the forward modelling operator and $\mathbf{y}$ is the measured data vector, $\lambda_{\mathrm{k}}$ the Lagrangian multiplier, and $\mathbf{C}$ is the $2^{\text {nd }}$ order smoothness matrix operator. The vector $\mathbf{d} \mathbf{x}_{\mathrm{k}}$ is added to the previous vector $\mathbf{x}_{\mathrm{k}}$ to obtain the updated resistivity parameters. The procedure is repeated until an acceptable misfit is reached between the measured and modelled data. The Lagrange multiplier is used to weight the model constraints against the data misfit and the strategy of decreasing $\lambda$ beginning from a starting value $(\lambda=1)$ down to a minimum value ( $\lambda=0.125$ ) was adopted. Furthermore the choice of calculating only the significant part of the Jacobian matrix was enabled in order to reduce the overall 3-D processing time. The algorithm converged to a final resistivity model after 8 iterations with an RMS less than 10\%, describing the subsurface resistivity up to the depth of 5 meters below the surface. Finally horizontal depth slices every 0.5 meter were extracted by the final 3-D inversion resistivity model. Specific sections of the slices were masked illustrate areas with no valid information due to the surface obstacles that prohibited the extension of the lines.

\subsection{Seismic Refraction Tomography}

The seismic refraction tomography survey covered the whole area of interest. Totally twenty four P-wave geophones were randomly installed on the ground following arbitrary directions in order to have a $3 \mathrm{D}$ coverage of the study area. The seismic energy was created by vertically striking a metal ground plate with a $7 \mathrm{Kg}$ sledgehammer (Figure 2-right). The shots in each location were repeated at least 4 times to ensure the good quality of signal by stacking the recorded waveforms. Totally 73 shots were used to collect about 1752 traveltimes $(73$ shots x 24 geophones $=1752$ raypaths). Prior the application of seismic tomography, all the collected seismic data were processed using the PickWIN (ver. 3.2.0.1) module of SeisImager processing software in order to pick the first arrivals, the traveltimes and the source - receivers location.

The data were afterwards pre-processed providing information about a) source and receiver exact locations including topographic corrections, b) the experimental geometry and c) the traveltimes recorded per source-receiver pair. Non-commercial software (ATOM_3D, Koulakov 2009) was 
used for the inversion and interpretation of the seismic refraction traveltime data. The program uses a ray tracing bending algorithm for solving the forward problem. The model parameterization can be applied by using nodes or cells, polygons or analytical laws. It is only necessary to define an initial velocity model of the study area. The raytracing starts from a straight line connecting source and receiver and iteratively (by using bending method) changes in order to finally achieve the minimum traveltime raypath. The calculated ray tends to travel through high velocity anomalies and avoids low velocity patterns. An iterative routine (LSQR- Paige and Saunders 1982) is used to solve the inverse seismic refraction problem. The amplitude and smoothness of the tomographic solution is controlled by applying regularization constraints. In order to reduce the effect of grid orientation, the inversion is applied in several differently oriented grids (i.e. $0^{\circ}$, $22^{\circ}, 45^{\circ}$ and $67^{\circ}$ ) and the results are stacked. The final velocity tomographic model was achieved after nine iterations and the final Root Mean Square (RMS) error was $1.03 \mathrm{~ms}$. Finally horizontal velocity tomograms every 0.5 meter up to the depth of 5.5 meters were extracted by the $3-\mathrm{D}$ velocity model.

\subsection{Ground Penetration Radar}

The GPR data were collected from an area of about 300 square meters with the Noggin Plus Smart Cart system using the $250 \mathrm{MHz}$ antennas (Figure 2-left) partly overlapping the southern part of the investigated area. The effort of the GPR survey was to map the vertical subsurface stratigraphy through parallel profiles. The distance between the individual lines was 0.5 meter while the resolution along each transect was 0.05 meters. This particular field set up can increase the spatial resolution of the subsurface reflections that are related to archaeological structures. The GPR sections were corrected for the local coordinate system. Then the first peak was estimated in order to define the initial useful signal from each line based on the intensity percentage of the first reflected wave (5-30\%). Specific filters (AGC, Dewow and DCshift) enhanced the reflected signal, while the rejection of the background noise and the data smoothing was accomplished by a trace-to-trace averaging filter. Finally, horizontal depth slices with thickness 0.2 meters were created by the original vertical sections assuming a velocity for the electromagnetic waves equal to $0.1 \mathrm{~m} / \mathrm{nsec}$, reaching the maximum depth of 2 meters below the ground.
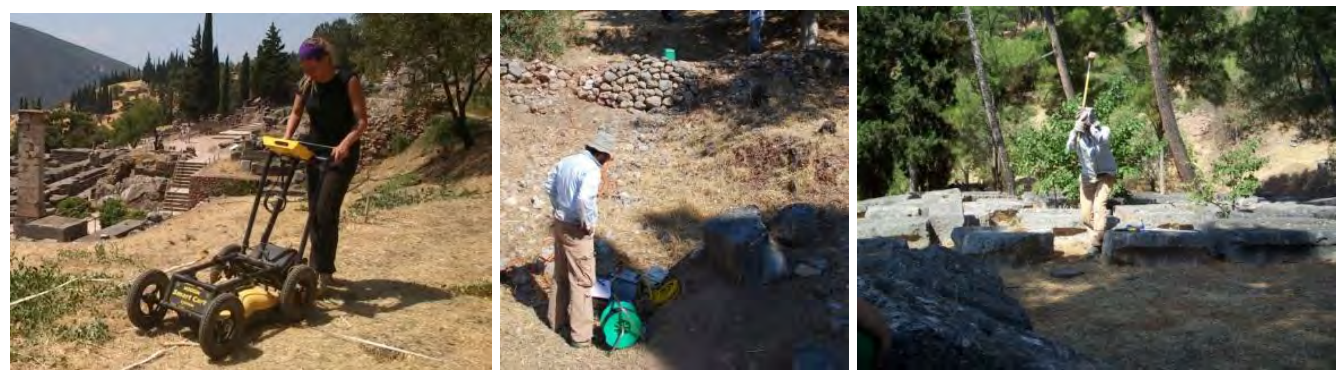

Figure 2 - Details of the geophysical methods with GPR (left), ERT (center) and SRT (right) that were applied in the area $A$ of the site.

\section{Integrated interpretation of geophysical results}

The results of the geophysical mapping are presented through colour scale maps depicting the horizontal and vertical variation of the ground resistivity and velocity of seismic waves, as well as the intensity of the reflected electromagnetic waves. The diverse applied geophysical techniques contributed in extracting horizontal slices that describe the spatial variation of the different physical properties in multiple depths below the ground. Reddish colours in the maps indicate increase of the physical property (resistivity, velocity and reflection amplitude) that can be potentially attributed to the existence of buried archaeological structure. The integrated and efficient interpretation of the geophysical results was made possible with the rectification of all the 
maps on a common coordinate system and the overlay of them on the topographic plan of the site through a Geographical Information System platform (Figures 3, 4, 5).

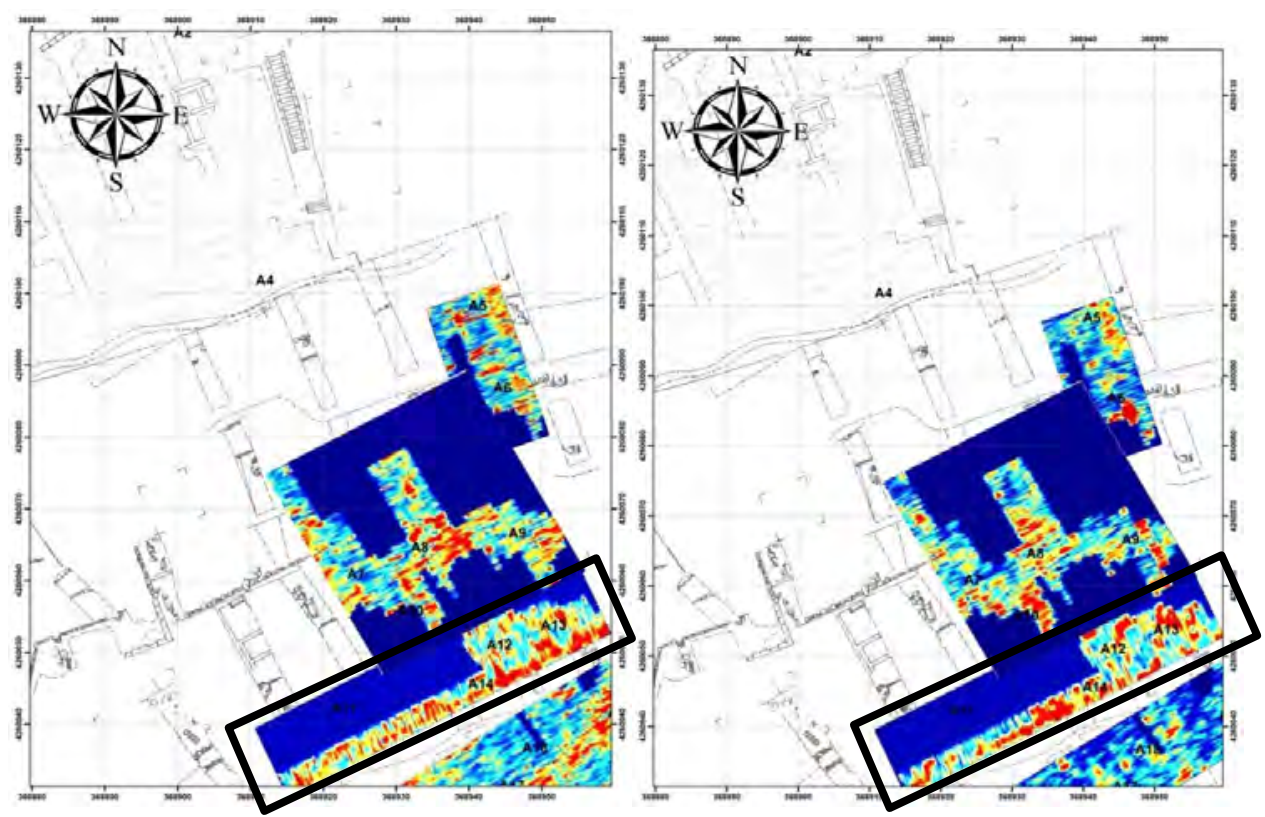

Figure 3 - GPR depth slices for depth less than 1 meter (left) and depth more than $1.5 \mathrm{~m}$ (right). The section of the area A that was surveyed with the GPR is depicted within the black rectangular.

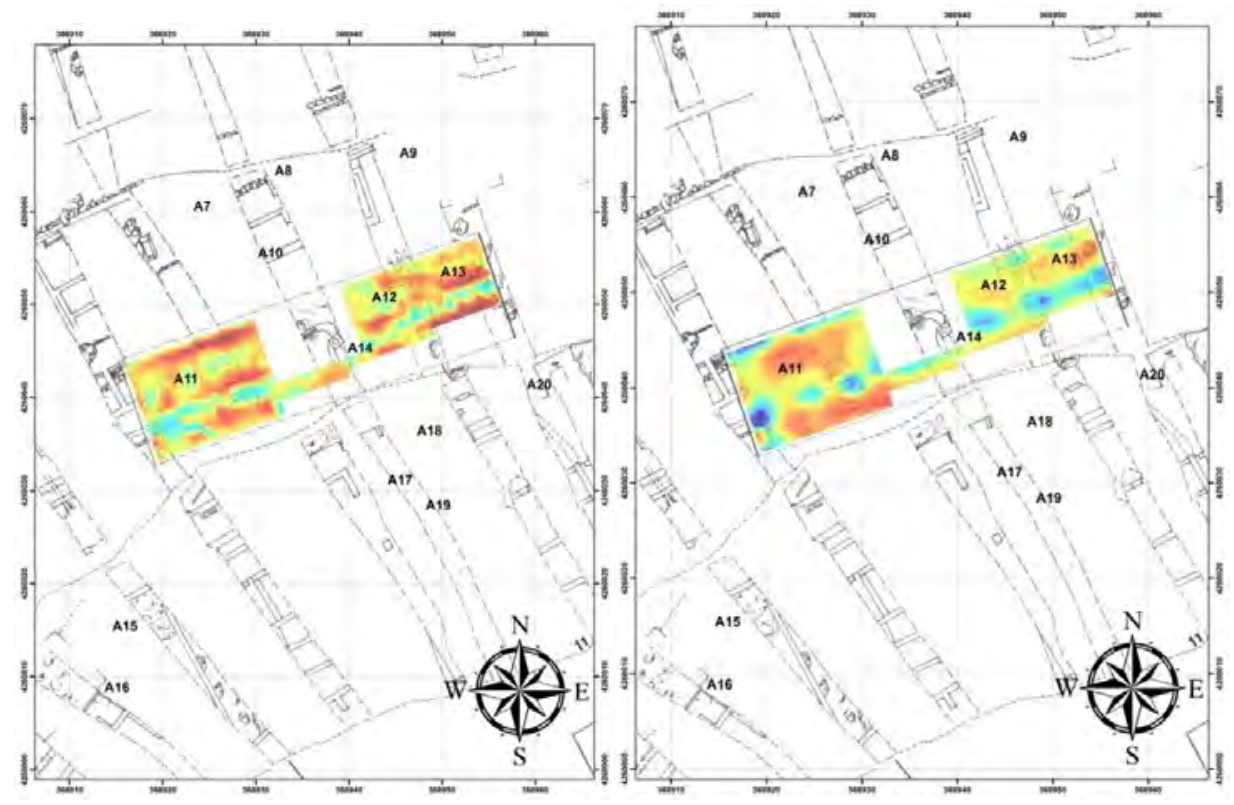

Figure 4 - ERT depth slices of the distribution of the soil resistivity of area A for depth 1 meter (left) and depth 2 meters (right). 

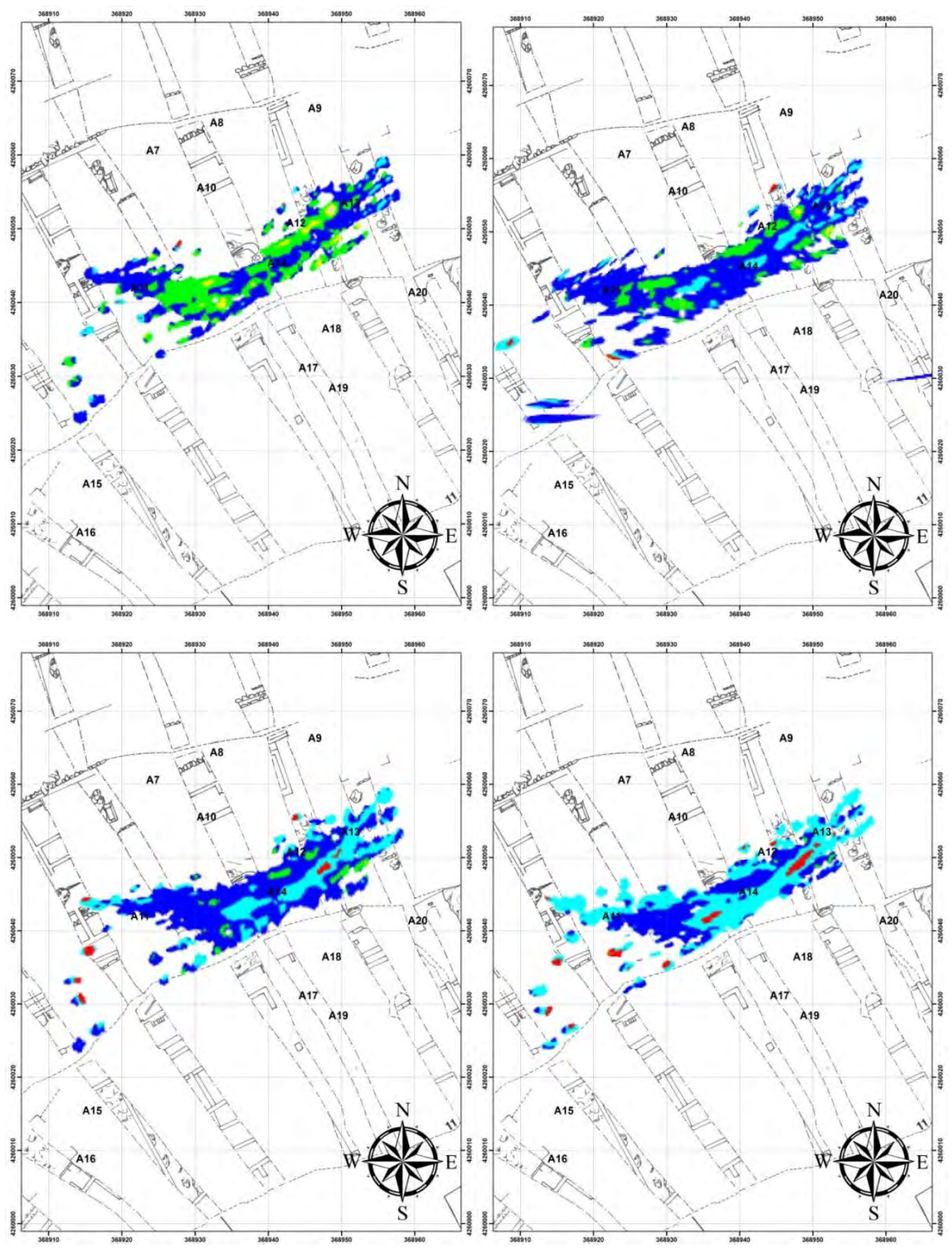

Figure 5 - Seismic depth slices for depth 1 meter (upper left), depth 2 meters (upper right), depth 3 meters (lower left) and depth 4 meters (lower right).

The results of all methods pinpointed to a number of anomalies. The ERT and GPR anomalies had the highest degree of correlation (Figures 3 and 4). The continuation of the wall structures to the east seems to be confirmed by the linear anomalies existing at A11. An elongated linear anomaly (A14) extending for more than 35 meters along the SW-NE direction seems to be consistently present in all datasets and may constitute another supporting wall (see also Figure 5). Towards the 
NE of this anomaly, two clusters of features appear at the location of A12 and A13, even if they are not clearly described. The GPR measurements indicate also the existence of vertical sections extending from the supporting wall, probably belonging to other architectural structures. Figure 6 presents the integrated diagrammatic interpretation of the geophysical anomalies resulted by all methods and different depth slices.

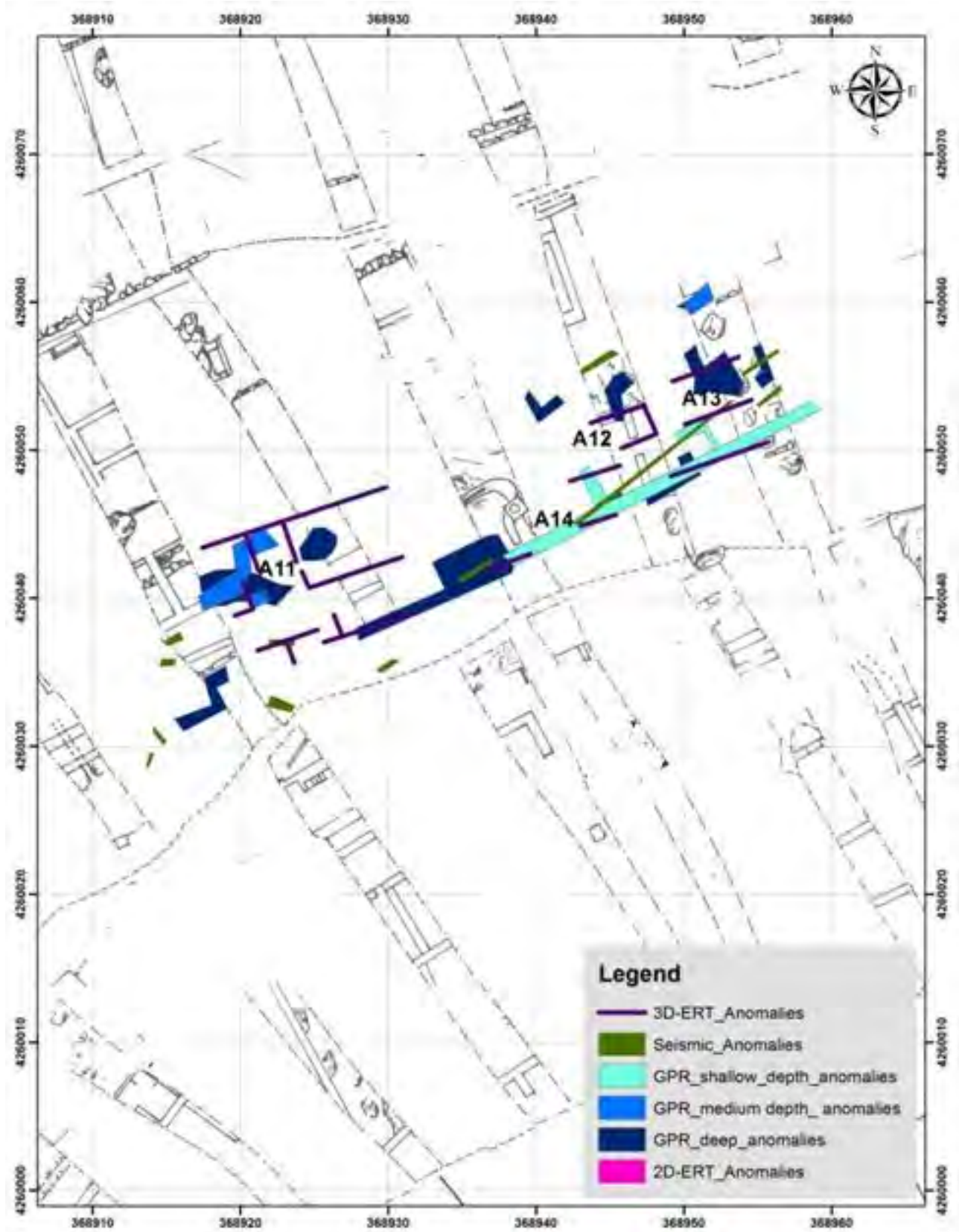

Figure 6 - integrated diagrammatic interpretation of the high resistivity, high velocity and high amplitude GPR reflection anomalies resulted by the interpretation of the ERT, SRT and GPR data that were collected in the area A of Delphi. 


\section{Conclusions}

The interpretation of electrical resistivity and seismic refraction tomographic geophysical data through inversion methods face the possibility to get different tomographic results from the same data file by applying different regularization or inversion parameters. This is caused by the geophysical ambiguity or uncertainty of geophysical interpretation, but also from the fact that each method is based on the measurements of different physical properties of the soil. Since in general, a unique solution cannot be reconstructed from a data set, geophysical interpretation is concerned either to determine properties of the subsurface that all possible solutions share, or to introduce assumptions to restrict the number of admissible solutions. In this sense a manifold geophysical field strategy has to be pursued (Sarris 2012).

The application of different tomographic techniques in the archaeological site of Delphi signifies the importance of the manifold geophysical strategy in order to extract the maximum subsurface information in a more efficient way. Each one of the methods applied has been able to suggest specific targets in terms of the physical quantity measured and the properties of the subsurface. The employment of different methods for the scanning of the site was valuable, since they provided complementary information and thus helped the delineation of the most significant features that were suggested by the various approaches.

\section{References}

Fong D. C.-L. and Saunders M.A. 2011. LSMR: An iterative algorithm for sparse least-squares problems, SIAM Journal of Scientific Computing, 33, 2950-2971.

Koulakov I. 2009. LOTOS code for local earthquake tomographic inversion, Benchmarks for testing tomographic algorithms, BSSA, Vol. 99(1) 194-214 doi: 10.1785/0120080013.

Luce J-M. 2012. Exploration du secteur Nord-Ouest de Delphes. La mission du 9 au 30 juillet 2012, unpublished report.

Paige C C and Saunders M A.1982. LSQR: An algorithm for sparse linear equations and sparse least squares, ACM trans. Math Soft., 8, 43-71.

Papadopoulos N.G., Tsourlos P., Tsokas G.N. and Sarris A. 2006. 2D and 3D Resistivity Imaging in Archaeological Site Investigation, Archaeological Prospection, 13, 3, 163-181.

Papadopoulos N.G., Tsourlos P., Papazachos C., Tsokas G.N., Sarris A. and Kim J.H. 2009. An Algorithm for the Fast 3-D Resistivity Inversion of Surface Electrical Resistivity Data: Application on Imaging Buried Antiquities, Geophysical Prospecting, 59, 557-575.

Sarris A. 2012. Multi+ or Manifold Geophysical Prospection? Computer applications and Quantitative methods in Archaeology, University of Southampton, 26-30 March 2012. 